\title{
High Glucose Levels Interferes the Endometrial Cancer Cell Response to Metformin Treatment over Time
}

\author{
Amanda Machado Weber*, Thomas Strowitzki and Ariane Germeyer \\ Department of Gynecological Endocrinology and Reproductive Medicine, Heidelberg University Women's Hospital, Heidelberg, Germany
}

*Corresponding author: Amanda Machado Weber, Department of Gynecological Endocrinology and Reproductive Medicine, Heidelberg University Women's Hospital, Heidelberg, Germany, Tel: +49 6221 56-7910; E-mail: amandabarrosm@yahoo.com.br

Received Date: September 26, 2018; Accepted Date: October 06, 2018; Published Date: October 10, 2018

Copyright: (c) 2018 Weber AM, et al. This is an open-access article distributed under the terms of the Creative Commons Attribution License, which permits unrestricted use, distribution, and reproduction in any medium, provided the original author and source are credited.

Citation: Weber AM, Strowitzki T, Germeyer A (2018) High Glucose Levels Interferes the Endometrial Cancer Cell Response to Metformin Treatment over Time. Br J Res Vol. 5 No. 3: 44.

\section{Abstract}

Endometrial cancer is one of the most common gynecological malignancies worldwide. Hyperinsulinemia and hyperglycemia seems to play a central role in endometrial carcinogenesis. Furthermore, diseases associate with insulin resistance and high blood glucose levels, as seen in polycystic ovary syndrome, obesity, and type II diabetes mellitus are considered as significant risk factors for the development and progression of type I endometrial cancer. Metformin treatment, an insulinsensitizing agent, has been suggested as therapy to inhibit cellular overgrowth and hyperplasia under these clinical conditions possibly preventing different types of cancer. This study evaluated in an environment with high glucose levels the population doubling behavior of endometrial cancer cells over time under hyperinsulinemic condition as well as therapeutic concentration of metformin in a longterm setting. The endometrial cancer cells not only showed resistance to metformin therapeutic dose but also respond to the treatment increasing drastically the cellular growth and aggressiveness in the presence of high glucose levels.

\section{Keywords: Hyperglycaemia; Hyperinsulinemia; Type I} endometrial cancer

\section{Introduction}

Endometrial Cancer (EC) is one of the most common gynecological malignancies worldwide and is classified into an estrogen-dependent type I and an estrogen independent type II form. Type $I$ is the most common EC, accounting for $75 \%-85 \%$ of all cases [1]. Hyperglycemia also contributes to the growth and carcinogenesis of endometrial cancer [2] and act as a critical link between the observed increased cancer risk in patients with type II diabetes [3]. Furthermore, diabetic women not only have a two-fold risk for endometrial cancer [4], but also demonstrate an increased cancer mortality rate compared to normoglycemic individuals [5]. Hyperinsulinemia seems to play a crucial role in endometrial carcinogenesis, consequently metformin, an insulin-sensitizing agent, is assumed to contribute to prevention of its development and induce reduction in the progression rate of EC. Numerous studies have indicated that metformin could be effective as an addition in cancer therapy along with its traditional role in treating diabetes [6-8], the beneficial effect of metformin in EC remains to be determined. Furthermore, metformin action is observed in experimental studies at suprapharmacological levels, short-term treatment and without estradiol addition, a known risk factor in endometrial proliferative disorders. In order to examine the effect of metformin treatment on EC development and metastatic potential recently we investigated the effect of low dose metformin $(0.1 \mathrm{mM})$ in an endometrial adenocarcinoma type I cell line [9]. To mimic insulin resistance, cells were treated in hyperinsulinemic environment exposed to normal glucose for 72 hours. As expected the migration and invasion ability of an endometrial cancer cell line in a high and normal glucose environment was increased by addition of insulin. A low metformin dose, however, was able to abolish this insulin effect in both conditions, leading to an equal migration and invasion as seen in the control. When insulin was not present metformin only reduced the migration capacity of EC cells in a normal glucose, but not in a hyperglycemic environment. These findings indicate that metformin even in low dose $(0.1 \mathrm{mM})$ has effective inhibitory effect on insulin actions in endometrial cancer cells. Furthermore, we found a reduction of migration in primary endometrial epithelial cells (eEP) in a high glucose environment, when lower metformin dose was present, while this effect was not visible in a hyperinsulinemic state, not in endometrial cancer cells. This finding suggests that endometrial cancer cells may be resistant to metformin, when glucose levels are elevated. While low dose metformin was unable to inhibit the proliferation alone or in the presence of insulin, a dose dependent metformin concentration curve $(0 \mathrm{mM}, 0.1 \mathrm{mM}, 1 \mathrm{mM}$ and $5 \mathrm{mM})$ proved the sensitivity of the cells to metformin in medium containing high as well as normal glucose levels for $72 \mathrm{~h}$. This effect, which was noted when at least $1 \mathrm{mM}$ metformin was present, was more pronounced in normal compared to high glucose environment.

According to our preliminary findings, we may say that physiological levels of metformin $(0.1 \mathrm{mM})$ may already inhibit the high cellular invasion and migration induced by insulin in 
endometrial cancers cells, even so it is ineffective to block the proliferation of these cells. Therefore, as far as we know, this is the first time that a direct anti-metastatic effect of metformin on insulin cancer progression action in endometrial tumor cells could be demonstrated. These results, lead us to evaluate in an environment with high glucose levels the cellular growth behavior of endometrial cancer cells over time under hyperinsulinemic condition as well as therapeutic concentration of metformin in a long-term setting.

\section{Materials and Methods}

\section{Cell culture}

The Ishikawa human adenocarcinoma cell line was purchased from Sigma-Aldrich (European Collection of Cell Culture authentication). Ishikawa cells were grown in MEM (SigmaAldrich, BY, Germany) supplemented with $5 \%$ fetal bovine serum (HyClone, Thermo Scientific, UT, USA), 1\% penicilin-streptomycin (Gibco), 1\% Non-essential Amino Acids (Sigma-Aldrich, BW, Germany), $5 \mathrm{og} / \mathrm{mL}$ bovine insulin (Sigma-Aldrich) at $37^{\circ} \mathrm{C}$ in a humidified atmosphere with $5 \% \mathrm{CO}_{2}$ until reach $80 \%$ confluence.

For mimic a high glucose environment the cells were cultured in the different groups with DMEM/F12 medium containing 17 $\mathrm{mM}$ glucose. The media of all conditions were supplemented with 10-8 M estradiol. The different conditions included: a) control: medium; b) Insulin: medium plus $100 \mathrm{ng} / \mathrm{mL}$ insulin; c) metformin: medium plus $0.1 \mathrm{mM}$ metformin; d) Insulin plus metformin: medium plus $100 \mathrm{ng} / \mathrm{mL}$ insulin and $0.1 \mathrm{mM}$ metformin. All chemicals were purchased from Sigma-Aldrich.

\section{Population doubling (PD) and Cumulative population doubling (CPD)}

To evaluate the cellular doubling behavior of an isolated endometrial cancer population through 20-days treatment, the cells were seeded into 24-wells plates at a density of $1 \times 10^{4}$ cells/ml in a Drug Free Medium (DFM) overnight. Cells were then treated with the indicated treatment (control; Insulin; metformin; Insulin plus metformin) for $72 \mathrm{~h}$. After this period, the cells were trypsinized, counted to determine the cellular density. Afterwards the cells from the respective group were seeded again at a density of $1 \times 10^{4}$ cells $/ \mathrm{ml}$ in a DFM overnight followed by treatment for $72 \mathrm{~h}$. In the day 4, 8, 12, 16 and 20 this protocol was performed and the cell number was determined. The PD was calculated as previously described using the equation $P D D[\log N(t)-\log N(t o)] / \log 2$, where $N(t)$ is the number of cells per well at the time of the count, and $N$ (to) is the initial number of cells. The CPD is the sum of the PDs over time [10].

\section{Analysis}

Descriptive data are represented as the mean \pm SEM of three independent experiments performed in triplicates. The PD and CPD are plotted versus the days of treatment.

\section{Results}

The response profile of an isolated population was evaluated by the CPD of endometrial cancer cell line through 20-days treatment. This assay allows us to check the cell growth and response to different acute and chronic treatments. The acute effect is determined from the PD, which considers the amount of times that the respective population has doubled in each 72 hours treatment interval. The chronic effect, the CPD is the sum of the PDs over the 20 days of treatment.

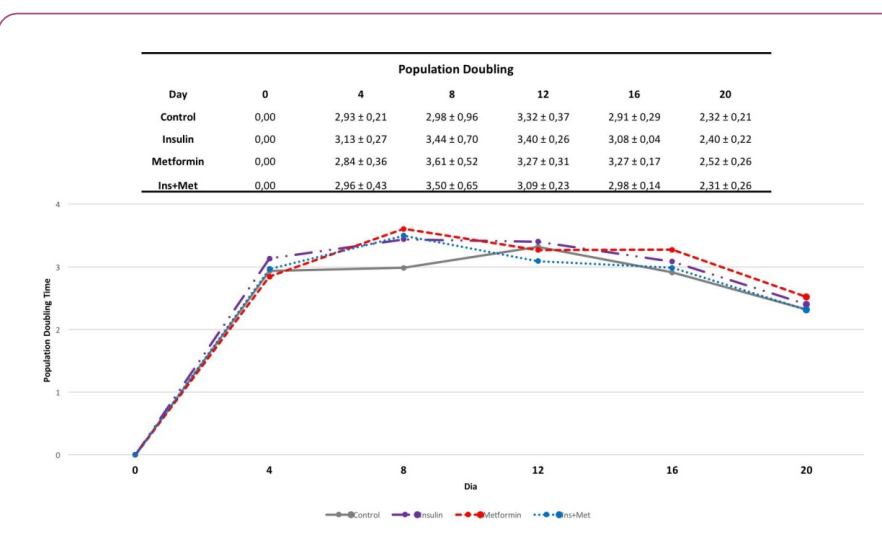

Figure 1 Population doubling of endometrial cancer cells through 20-days treatment environment with high glucose levels. Day 0, 4, 8, 12, 16 and 20 are the counting points. Descriptive data represented as the mean \pm SEM.

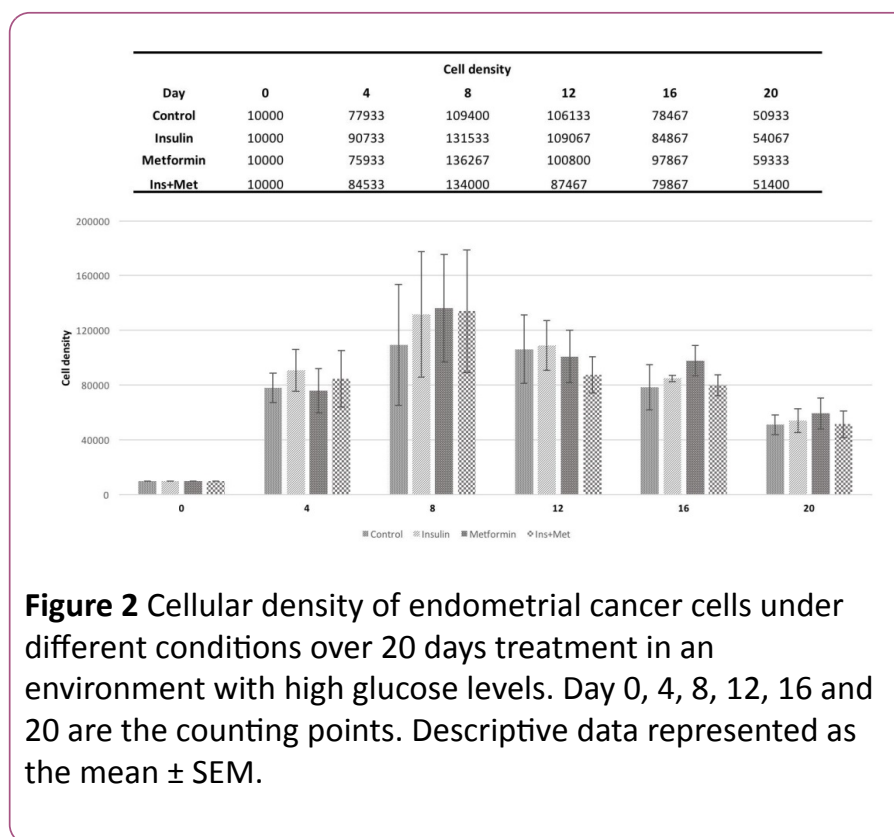

The Figure 1 shows the effect of the different treatments on the amount of times that the EC cells doubled over each 72 hours treatment. In the first 72 hours, the cells treated with insulin only and the cells treated with insulin plus metformin showed the highest cellular density (Figure 2), exhibiting a doubling time of 3,13-fold and 2,97-fold, respectively, followed by control group 2,93-fold. Moreover, the cells treated with metformin only showed the lowest cellular growth compared to the other groups, a PD of 2,84-fold. However, the PD pattern of 
endometrial cancer cells over time changed, where in the last two counting points the cells treated with metformin or insulin only showed the highest PD compared to the other groups. When we evaluated the effect of chronic treatment through 20 days using the CPD, in the first 3 time points, the cells treated with insulin showed the highest cellular growth pattern compared to the other groups (Figure 3).

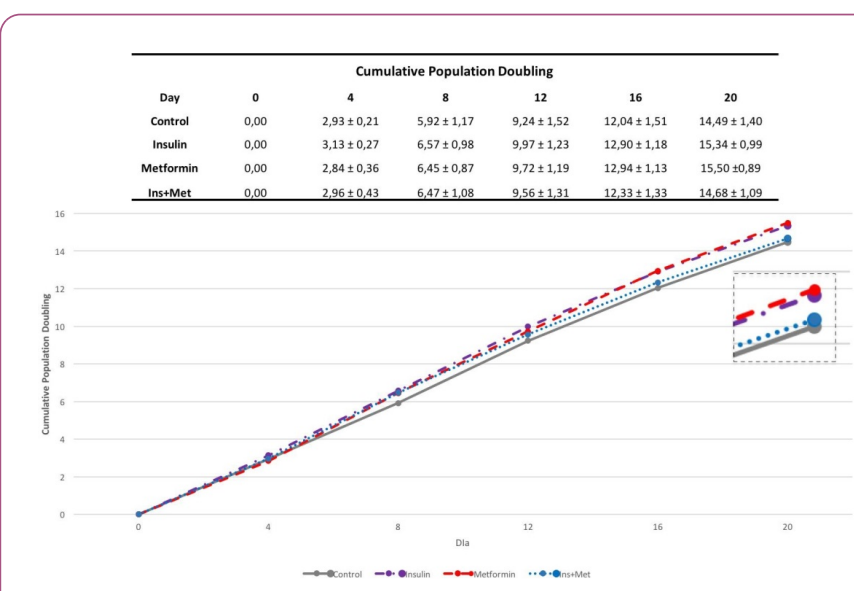

Figure 3 Cumulative population doubling of endometrial cancer cells under different condition in an environment with high glucose levels over 20 days treatment. Day 0, 4, 8, 12, 16 and 20 are the counting points. Descriptive data represented as the mean \pm SEM.

However, the cells treated with metformin only showed in the beginning the lowest growth rate, being this pattern altered in each new count point over the treatment time. In the second point (day 8), the cells treated with metformin only already showed one CPD of 6,45-fold, whereas 5,92-fold in the control group. In the next point (day 12), this cellular population showed higher CPD compared to the cells treated with insulin plus metformin as well as control cells. Furthermore, in the last 2 time points (day 16 and day 20), the cells treated with metformin only overcome the CPD of all other groups, including the cells treated with insulin only. Showing a CPD of 15,50-fold, followed by the cells treated with insulin, the cells that received co-treatment and the control group.

\section{Discussion and Conclusion}

In this study we evaluate the effect of different EC risk factors conditions on EC cell growth behavior as well as the effect of metformin therapeutic dose over time. We were able to see the effect of the different conditions on a specific cellular population, as over the counting and seeding points; a natural population selection is performed. While the metformin therapeutic dose showed a possible trend to inhibit EC cells growth during acute treatment, through 20 days treatment the cells treated with this drug showed the highest population doubling, overcoming the cells treated with insulin. Considering our preview findings [9], which we showed that high glucose levels may create an optimal environment for EC cells exhibit resistance to low metformin dose, we assume that over the counting points in an environment surrounded by high glucose levels, the EC cells not only showed resistance to metformin therapeutic dose but also responded to the treatment increasing drastically the cellular growth and aggressiveness. Nevertheless, our findings demonstrate the importance of using glucose metabolism therapeutic targets as potential strategy to EC prevention and treatment. Reinforcing the possibility of using metformin as an adjuvant agent, as metformin in vivo acts indirectly decreasing glucose and insulin levels, making the environment more favorable for its action directly in the cells.

\section{Conflict of Interest}

All authors disclose no conflict of interest.

\section{References}

1. Garg K, Soslow RA (2014) Endometrial carcinoma in women aged 40 years and younger. Archives of pathology and laboratory medicine 138: 335-342.

2. Lambe M, Wigertz A, Garmo H, Walldius G, Jungner I, et al. (2011) Impaired glucose metabolism and diabetes and the risk of breast, endometrial, and ovarian cancer. Cancer causes and control: CCC 22: 1163-1171.

3. Szablewski L (2014) Diabetes mellitus: influences on cancer risk. Diabetes/metabolism research and reviews 30: 543-553.

4. Friberg E, Orsini N, Mantzoros CS, Wolk A (2007) Diabetes mellitus and risk of endometrial cancer: a meta-analysis. Diabetologia 50: 1365-1374.

5. Barone BB, Yeh HC, Snyder CF, Peairs KS, Stein KB, et al. (2008) Long-term all cause mortality in cancer patients with preexisting diabetes mellitus: a systematic review and meta-analysis. JAMA 300: 2754-2764.

6. Umene K, Banno K, Kisu I, Yanokura M, Nogami Y, et al. (2013) New candidate therapeutic agents for endometrial cancer: potential for clinical practice (review). Oncology reports 29: 855-860.

7. Febbraro T, Lengyel E, Romero IL (2014) Old drug, new trick: repurposing metformin for gynecologic cancers? Gynecologic oncology 135: 614-621.

8. He H, Ke R, Lin H, Ying Y, Liu D, et al. (2015) Metformin, an old drug, brings a new era to cancer therapy. Cancer journal 21: 70-74.

9. de Barros Machado A, dos Reis V, Weber S, Jauckus J, Brum I, et al. (2016) Proliferation and metastatic potential of endometrial cancer cells in response to metformin treatment in a high versus normal glucose environment. Oncology Letters 12: 3626-3632.

10. Zamin LL, Filippi-Chiela EC, Dillenburg-Pilla P, Horn F, Salbego C, et al. (2009) Resveratrol and quercetin cooperate to induce senescence-like growth arrest in C6 rat glioma cells. Cancer Sci 100: $1655-1662$ 